\title{
G2019S Mutation in the Leucine-Rich Repeat Kinase 2 Gene Is Not Associated with Multiple System Atrophy
}

\author{
Laurie J. Ozelius, $\mathrm{PhD},{ }^{1} *$ Tatiana Foroud, $\mathrm{PhD},{ }^{2}$ Susanne May, $\mathrm{PhD},{ }^{3,4}$ Geetha Senthil, $\mathrm{PhD},{ }^{1}$ \\ Paola Sandroni, MD, PhD, ${ }^{5}$ Phillip A. Low, MD, ${ }^{5}$ Stephen Reich, MD, ${ }^{6}$ Amy Colcher, MD, ${ }^{7}$ \\ Matthew B. Stern, MD, ${ }^{7}$ William G. Ondo, MD, ${ }^{8}$ Joseph Jankovic, MD, ${ }^{8}$ Neng Huang, MD, ${ }^{9}$ \\ Caroline M. Tanner, MD, PhD, ${ }^{9}$ Peter Novak, MD, PhD ${ }^{10}$ Sid Gilman, MD, FRCP,${ }^{11}$ \\ Frederick J. Marshall, MD, ${ }^{12}$ G. Frederick Wooten, MD ${ }^{13}$ Thomas C. Chelimsky, MD, ${ }^{14}$ \\ Clifford W. Shults, MD, ${ }^{3,15}$ and The North American Multiple System Atrophy Study Group \\ ${ }^{I}$ Department of Molecular Genetics, Albert Einstein College of Medicine, Bronx, New York, USA \\ ${ }^{2}$ Department of Medical and Molecular Genetics, Indiana University, Indianapolis, Indiana, USA \\ ${ }^{3}$ Department of Neurosciences, University of California, La Jolla, San Diego, California, USA \\ ${ }^{4}$ Department of Family and Preventive Medicine, University of California, La Jolla, San Diego, California, USA \\ ${ }^{5}$ Department of Neurology, Mayo Clinic, Rochester, Minnesota, USA \\ ${ }^{6}$ Department of Neurology, School of Medicine, University of Maryland, Baltimore, Maryland, USA \\ ${ }^{7}$ Parkinson's Disease and Movement Disorders Center, Pennsylvania Hospital, Philadelphia, Pennsylvania, USA \\ ${ }^{8}$ Department of Neurology, Baylor College of Medicine, Houston, Texas, USA \\ ${ }^{9}$ Parkinson's Institute, Sunnyvale, California, USA \\ ${ }^{10}$ Department of Neurology, Boston University, Boston, Massachusetts, USA \\ ${ }^{11}$ Department of Neurology, University of Michigan, Ann Arbor, Michigan, USA \\ ${ }^{12}$ Department of Neurology, University of Rochester, Rochester, New York, USA \\ ${ }^{13}$ Department of Neurology, University of Virginia Health System, Charlottesville, Virginia, USA \\ ${ }^{14}$ Department of Neurology, Case Western Reserve University, Cleveland, Ohio, USA \\ ${ }^{15}$ Veterans Affairs San Diego Healthcare System, San Diego, California, USA
}

\begin{abstract}
Multiple system atrophy (MSA) is characterized clinically by Parkinsonism, cerebellar dysfunction, and autonomic impairment. Multiple mutations in the LRRK2 gene are associated with parkinsonian disorders, and the most common one, the G2019S mutation, has been found in $\sim 1 \%$ of sporadic cases of Parkinsonism. In a well-characterized cohort of 136
\end{abstract}

subjects with probable MSA and 110 neurologically evaluated control subjects, none carried the G2019S mutation. We conclude that the G2019S mutation in the LRRK2 gene is unlikely to be associated with MSA. (C) 2007 Movement Disorder Society

Key words: multiple system atrophy; LRRK2; Parkinsonism.
Multiple system atrophy (MSA) is a progressive, degenerative, neurological disease, which is characterized by Parkinsonism, cerebellar dysfunction, and autonomic

*Correspondence to: Laurie Ozelius, Albert Einstein College of Medicine, Molecular Genetics, Ull 1211, 1300 Morris Park Ave., Bronx, NY 10461. E-mail: Ozelius@aecom.yu.edu

Received 16 October 2006; Accepted 17 October 2006

Members of the North American Multiple System Atrophy Study Group are listed in the Appendix

Published online 17 January 2007 in Wiley InterScience (www. interscience.wiley.com). DOI: 10.1002/mds.21343 failure. ${ }^{1,2}$ The pathological hallmark of the disorder is the presence of glial cytoplasmic inclusions (GCI) in oligodendrocytes, and a major component of GCI is $\alpha$-synuclein. ${ }^{3} \mathrm{~A}$ number of disorders characterized by inclusions containing $\alpha$-synuclein have been grouped together as synucleinopathies, and these diseases include MSA, Parkinson's disease (PD), and Lewy body dementia. ${ }^{4}$

In 2004, two groups independently reported mutations in the gene leucine-rich repeat kinase 2 (LRRK2) in families with autosomal dominant PD mapping to the 
PARK8 locus. 5,6 As a number of the families involved are of Basque-descent, Paisan-Ruiz et al..$^{5}$ named the protein involved dardarin, derived from the Basque word dardara meaning tremor. Zimprich et al. ${ }^{6}$ reported that the common pathological feature in cases with mutations in this gene was neuronal loss and gliosis in the substantia nigra. Two cases were noted to have Lewy bodies (one relatively limited to the brainstem and one with widespread Lewy bodies). However, pleiomorphic pathology was also reported, including one case with progressive supranuclear palsy-like pathology and one kindred with clinical features of motor neuron disease, a mild decrease in anterior horn cells, gliosis, and axonal spheroids. Within months of these reports, a number of groups identified a specific mutation, G2019S $(6055 \mathrm{G} \rightarrow$ A nucleotide change), not found in the initial studies, and it is the most common mutation found to date in familial 7,8 and sporadic PD $^{9}$ in European and North American populations. The prevalence of this mutation in PD patients varies with ethnicity. It has been reported to be low in Asians, ${ }^{10}$ but high in North African Arabs ${ }^{11}$ and Ashkenazi Jews. ${ }^{12}$ The penetrance of this mutation appears to increase with age, and it may not be fully penetrant. ${ }^{13}$

Because of the relatively high frequency of the LRRK2 G2019S mutation in sporadic PD and the observation that mutations in this gene can be associated with atypical parkinsonian disorders, we determined the frequency of this mutation in a carefully characterized cohort of MSA patients and case-control subjects.

\section{SUBJECTS AND METHODS}

The study, which is part of an ongoing study of environmental and genetic risk factors for MSA, was conducted in accordance with the Declaration of Helsinki. Subjects were enrolled at 11 sites in the United States: University of California, San Diego; Parkinson's Institute; Mayo Clinic; University of Michigan; Case Western Reserve University; Baylor College of Medicine; University of Virginia; University of Maryland; University of Pennsylvania; University of Rochester; Boston University. The protocol was approved by the Institutional Review Board at each site and the Albert Einstein College of Medicine, and the subjects were enrolled after the study had been carefully explained and the subjects had signed an informed consent. Two hundred and fortysix subjects were evaluated for the mutation: 136 MSA patients and 110 control subjects. All individuals were evaluated with a medical history, neurological examination, and the Unified MSA Rating Scale. ${ }^{14}$ The neurologists evaluating the subjects had all participated in a validation process to ensure consistent evaluation of the subjects and application of the Unified MSA Rating Scale. All MSA subjects were diagnosed with probable MSA according to the Consensus Criteria. ${ }^{15}$ The MSA subjects were classified as having the parkinsonian variant of MSA (MSA-P), if the symptoms of Parkinsonism developed a year or more before cerebellar dysfunction, or the cerebellar variant of MSA (MSA-C), if the symptoms of cerebellar dysfunction developed a year or more before Parkinsonism. If the data did not clearly indicate whether Parkinsonism or cerebellar dysfunction occurred first, then the MSA subjects were defined as mixed (MSA-mixed). The control subjects exhibited no symptoms or signs indicative of MSA. Designation of ethnicity and race was based on self-reporting. Each subject was assigned an anonymous ID code. Blood was drawn from all participants.

DNA was extracted from white blood cells using the Puregene procedure (Gentra Systems, Minneapolis, MN). The G2019S mutation in LRRK2 was genotyped by Pyrosequencing (Biotage, Uppsala, Sweden) using the methods and primers described. ${ }^{12}$

\section{RESULTS}

The mean age at disease onset in the MSA subjects was $58.1 \pm 9.3$, which is similar to that in other series. ${ }^{2,16,17}$ Sixty percent of the MSA subjects were men, which is consistent with previous reports. ${ }^{2,17}$ More than half $(57 \%)$ of the subjects had the MSA-P form, with the remaining samples split about equally between MSA-C $(22 \%)$ and MSA-mixed (21\%). The frequency of MSA-P in this cohort is similar to that described in European MSA cases. ${ }^{2,18}$ The vast majority of our MSA subjects were white $(94 \%)$, and this may reflect the referral patterns of the 11 enrolling centers. The data are summarized in Table 1.

We did not find the G2019S mutation in the LRRK2 gene in any of our 136 MSA subjects or 110 control subjects. Our findings are consistent with previous studies of control cohorts. ${ }^{19,20}$

\section{DISCUSSION}

This is the first screening of a large cohort of MSA patients for the G2019S mutation in the LRRK2 gene. The G2019S mutation is found in about $1 \%$ of sporadic PD patients. ${ }^{9}$ If this mutation were found at a similar frequency in MSA as is observed in sporadic PD, we would expect 1 to 2 of our MSA subjects to carry the G2019S mutation, but we did not observe this mutation in any of our cases. Although our power to detect a mutation frequency substantially lower than this is limited, it is clear from our data that this mutation is not a major risk factor for MSA. A recent study of 14 muta- 
TABLE 1. Demographic information for MSA patients and control subjects

\begin{tabular}{lcc}
\hline & Control & MSA \\
\hline Number of subjects & 110 & 136 \\
Age & $64.1 \pm 8.7$ & $62.7 \pm 8.5$ \\
Age at onset & NA & $58.1 \pm 9.3$ \\
Sex & & \\
$\quad$ Male & $46(42)^{a}$ & $81(60)$ \\
Female & $64(58)$ & $55(40)$ \\
Race & $107(97)$ & $128(94)$ \\
White & 1 & 6 \\
Asian & 1 & 0 \\
African American & 1 & 0 \\
Pacific Islander & & 2 \\
Other or & 0 & 729 \\
unspecified & & $77(57)$ \\
Ethnicity & 107 & $30(22)$ \\
Not Hispanic & 3 & $29(21)$ \\
Hispanic & & 0 \\
Classification of MSA & NA & \\
MSA-P & NA & \\
MSA-C & NA & \\
MSA-mixed & 0 & \\
LRK2-G2019S & & \\
\hline
\end{tabular}

${ }^{a}$ Values in parentheses indicate percentages.

tions in the LRRK2 gene in parkinsonian subjects from Singapore did not find any mutations in the gene in 15 MSA subjects. ${ }^{10}$ Ross et al. ${ }^{20}$ did not detect the G2019S mutation in 43 pathologically confirmed cases of MSA. The absence of the G2019S mutation in control subjects is similar to that reported by other groups. ${ }^{19-21}$ The G2019S mutation is infrequent in Alzheimer's disease, progressive supranuclear palsy, and frontotemporal dementia. ${ }^{20,22-24}$

It should be noted that the cases initially reported by Zimprich et al. ${ }^{6}$ to have pathology not typical of PD were associated with the Y1699C and R1441G mutations in the LRRK2 gene. However, studies of the pathology of cases with the G2019S mutation ${ }^{20,25}$ have revealed classical changes of PD with Lewy bodies, but also changes of PD without Lewy bodies. In some cases, senile plaques and neurofibrillary tangles in the cortex were also found.

LRRK2 is encoded by 51 exons and is an unusually large protein with 2,527 amino acids. ${ }^{12}$ It has a number of functional domains including two enzymatic regions: ankyrin repeat region, leucine-rich repeat domain, Ras of complex, C terminal of Ras, protein kinase, and WD40 domain. It is conceivable that mutations in other regions of this complex and multifunctional molecule may be associated with the Parkinsonism and synuclein inclusions of MSA. However, we have carefully reviewed the reports of LRRK2 mutations other than G2019S in parkinsonian disorders, and found that they have typically been found in only one or a few families, or in isolated populations. A recent screen of 458 patients with lateonset PD or Parkinson's plus syndromes (including 15 cases of MSA) for 14 mutations in the LRRK2 gene found a single PD case with an R1441C mutation $(0.26 \%) .{ }^{10}$ Given the rarity of mutations other than G2019S, it is unlikely that these very rare mutations occur in our cohort, which did not include familial cases, and was recruited throughout the United States.

In summary, our screening of the largest cohort of patients with probable MSA reported to date indicated that the G2019S mutation in the LRRK2 gene is unlikely to be associated with the disorder.

\section{APPENDIX}

The following members of the North American Multiple System Atrophy Study Group participated in this study and contributed toward this report:

\section{The North American Multiple System Atrophy Study Group Steering Committee}

University of California at San Diego: Clifford W. Shults, M.D., Eliezer Masliah., M.D., Susanne May, Ph.D.; University of Michigan: Sid Gilman, M.D.; Parkinson's Institute: Caroline Tanner, M.D., Ph.D.; University of Washington: Walter Kukull, Ph.D.; University of Pennsylvania: Virginia Lee, Ph.D., John Trojanowski, M.D., Ph.D.; Mayo Clinic, Rochester: Phillip Low, M.D.; University of Rochester: Ira Shoulson, M.D.; Albert Einstein College of Medicine: Laurie Ozelius, Ph.D.; Indiana University: Tatiana Foroud, Ph.D.

\section{Study Coordinators}

Debra Berry, University of Rochester; Marsha Burks, University of Michigan; Nancy Zappala, University of Maryland; Toni Gehrking, Mayo Clinic; Melissa Diggin, Boston University; Ernesto Jimenez, Baylor College of Medicine; Kathleen Comyns, Parkinson's Institute; Robert Davis, University of Virginia Health System; Mary Lloyd, Pennsylvania Hospital; Deborah Fontaine, University of California at San Diego.

\section{Biostatistics}

Susanne May, Devon Gessert, and B. Brooke Sowell, University of California at San Diego.

Acknowledgements: The work was supported by NIH grant NS P01 044233 and the William M. Spencer, Jr. Research Fund.

\section{REFERENCES}

1. Quinn N, Wenning G. Multiple system atrophy. Curr Opin Neurol 1995;8:323-326.

2. Wenning GK, Ben Shlomo Y, Magalhaes M, Daniel SE, Quinn NP. Clinical features and natural history of multiple system atrophy. An analysis of 100 cases. Brain 1994;117:835-845.

3. Dickson DW, Lin W, Liu WK, Yen SH. Multiple system atrophy: a sporadic synucleinopathy. Brain Pathol 1999;4:721-732.

4. Galvin JE, LeeVM, Trojanowski JQ. Synucleinopathies: clinical and pathological implications. Arch Neurol 2001;58:186-190. 
5. Paisan-Ruiz C, Jain S, Evans EW, et al. Cloning of the gene containing mutations that cause PARK8-linked Parkinson's disease. Neuron 2004;44:595-600.

6. Zimprich A, Biskup S, Leitner P, et al. Mutations in LRRK2 cause autosomal-dominant parkinsonism with pleomorphic pathology. Neuron 2004;44:601-607.

7. Di Fonzo A, Rohe CF, Ferreira J, et al. A frequent LRRK2 gene mutation associated with autosomal dominant Parkinson's disease. Lancet 2005;365:412-415.

8. Nichols WC, Pankratz N, Hernandez D, et al. Genetic screening for a single common LRRK2 mutation in familial Parkinson's disease. Lancet 2005;365:410-412.

9. Gilks WP, Abou-Sleiman PM, Gandhi S, et al. A common LRRK2 mutation in idiopathic Parkinson's disease. Lancet 2005;365:415416.

10. Tan EK, Skipper L, Chua E, et al. Analysis of 14 LRRK2 mutations in Parkinson's plus syndromes and late-onset Parkinson's disease. Mov Disord 2006;21:997-1001.

11. Lesage S, Durr A, Tazir M, et al. LRRK2 G2019S as a cause of Parkinson's disease in North African Arabs. N Engl J Med 2006; 354:422-423.

12. Ozelius LJ, Senthil G, Saunders-Pullman R, et al. LRRK2 G2019S as a cause of Parkinson's disease in Ashkenazi Jews. N Engl J Med 2006;354:424-425.

13. Kachergus J, Mata IF, Hulihan M, et al. Identification of a novel LRRK2 mutation linked to autosomal dominant parkinsonism: evidence of a common founder across European populations. Am J Hum Genet 2005;76:672-680.

14. Wenning GK, Tison F, Seppi K, et al.; Multiple System Atrophy Study Group. Development and validation of the Unified Multiple System Atrophy Rating Scale (UMSARS). Mov Disord 2004;19: 1391-1402.

15. Gilman S, Low PA, Quinn N, et al. Consensus statement on the diagnosis of multiple system atrophy. J Neurol Sci 1999;163:9498.
16. Testa D, Monza D, Ferrarini M, Soliveri P, Girotti F, Filippini G. Comparison of natural histories of progressive supranuclear palsy and multiple system atrophy. Neurol Sci 2001;22:247-251.

17. Watanabe H, Saito Y, Terao S, et al. Progression and prognosis in multiple system atrophy: an analysis of 230 Japanese patients. Brain 2002;125:1070-1083.

18. Geser F, Seppi K, Stampfer-Kountchev M, et al. The European Multiple System Atrophy-Study Group (EMSA-SG). J Neural Transm 2005;112:1677-1686.

19. Kay DM, Zabetian CP, Factor SA, et al. Parkinson's disease and LRRK2: frequency of a common mutation in U.S. movement disorder clinics. Mov Disord 2006;21:519-523.

20. Ross OA, Toft M, Whittle AJ, et al. Lrrk2 and Lewy body disease. Ann Neurol 2006;59:388-393.

21. Paisan-Ruiz C, Lang AE, Kawarai T, et al. LRRK2 gene in Parkinson disease: mutation analysis and case control association study. Neurology 2005;65:696-700.

22. Hernandez D, Paisan-Ruiz C, Crawley A, et al. The dardarin G 2019 S mutation is a common cause of Parkinson's disease but not other neurodegenerative diseases. Neurosci Lett 2005;389:137139.

23. Saunders-Pullman R, Lipton RB, Senthil G, et al. Increased frequency of the LRRK2 G2019S mutation in an elderly Ashkenazi Jewish population is not associated with dementia. Neurosci Lett 2006;402:92-96.

24. Toft M, S.B. Sando SB, S. Melquist S, et al. LRRK2 mutations are not common in Alzheimer's disease. Mech Ageing Dev 2005;126: 1201-1205.

25. Giasson BI, Covy JP, Bonini NM, et al. Biochemical and pathological characterization of Lrrk2. Ann Neurol 2006;59:315-322.

26. Mata IF, Wedemeyer WJ, Farrer MJ, Taylor JP, Gallo KA. LRRK2 in Parkinson's disease: protein domains and functional insights. Trends Neurosci 2006;29:286-93. 